\title{
Non-nociceptive pain in rheumatoid arthritis is frequent and affects disease activity estimation: cross-sectional data from the FRAME study
}

\begin{tabular}{lccc}
\multicolumn{4}{c}{ Supplementary table A: Differences between participants with high and low PDQ-scores } \\
& $\begin{array}{c}\text { PDQ score }<13 \\
(\mathrm{n}=66)\end{array}$ & $\begin{array}{c}\text { PDQ score } \geq 13 \\
(\mathrm{n}=35)\end{array}$ & p-value \\
\hline Female (\%) & $45(68.2)$ & $31(88.57)$ & 0.03 \\
Age, years (SD) & $56.1(14.7)$ & $52.1(17.9)$ & 0.24 \\
Body mass index (SD) & $25.8(4.7)^{++}$ & $26.9(5.8)$ & 0.32 \\
Disease duration, months & $15.5(1-104)$ & $50(15-147)$ & 0.04 \\
Current smoker (\%) & $11(16.9)^{+}$ & $9(24.7)$ & 0.29 \\
Corticosteroid usage (\%) & $11(16.7)$ & $5(14.3)$ & 0.76 \\
28 Swollen joint count & $2(1-7)$ & $3(2-7)$ & 0.24 \\
28 Tender joint count & $5(3-10)$ & $12(7-16)$ & $<.0001$ \\
Tender point count, 0-18 & $6(4-14)$ & $11(7-16)$ & 0.005 \\
$\geq 11$ tender points (\%) & $20(30.3)$ & $18(51.4)$ & 0.04 \\
DAS28 (SD) & $4.2(1.1)$ & $4.9(1.0)$ & 0.002 \\
DAS28-P, 0-1 (SD) & $0.47(0.13)$ & $0.57(0.09)$ & $<.0001$ \\
HAQ-DI, 0 - 3 & $0.75(0.38-1.25)$ & $1.38(0.88-1.88)$ & 0.0001 \\
VAS-fatigue, mm & $53.5(27-71)$ & $74(58-89)$ & 0.0005 \\
VAS-pain, mm & $42(24-60)$ & $69(50-81)$ & $<.0001$ \\
VAS-global health, mm & $56(32-77)$ & $73(55-88)$ & 0.01 \\
GAD-10 score, 0 - 50 & $6(3-10)^{+}$ & $10(6-14)$ & 0.002 \\
MDI score, 0 - 50 & $8(4-12)^{+}$ & $12(7-21)$ & 0.003 \\
SF-36 PCS, 0 - 100 & $34.8(28.7-42.0)$ & $30.3(24.4-35.4)$ & 0.02 \\
SF-36 MCS, 0 - 100 & $51.0(40.2-56.6)$ & $41.7(30.8-50.2)$ & 0.001 \\
CRP, mg / mL & $8(3-15)$ & $3(0.5-15)$ & 0.06 \\
IgM-RF positive (\%) & $41(62.1)$ & $23(65.7)$ & 0.72 \\
USD score, 0 - 72 & $9(5-15)$ & $10(4-14)$ & 0.91 \\
RAMRIS synovitis $(0-21)^{\#}$ & $7.5(5-10)$ & $9(6.5-11)$ & 0.10 \\
\hline
\end{tabular}

Values are median (25th, 75th percentiles) unless specified otherwise. Differences between groups were tested using the t-test or the Mann -Whitney U test for continuous data and chi-square test for categorical data.

CRP, C-reactive protein; DAS28, Disease activity score based on the 28 joint count; DAS28-P, proportion of the DAS28-score that pertains from the patient reported subcomponents; GAD-10, Generalised anxiety questionnaire; HAQ-DI, Health assessment questionnaire disability index; MDI, Major depression inventory; PQD, painDETECT questionnaire; RF, Rheumatoid factor; SF36 MCS, Short form-36 mental component summary score; SF-36 PCS, Short form-36 physical component summary score; VAS, Visual analogue scale.

${ }^{\#}$ Wrist plus MCP. Due to contraindications or participant refusal 26 patients did not receive a MRI scan. Distributions were as following: PDQ-score <13: $n=50$, PDQ-score >18: $n=24$.

${ }^{+}$one missing observation

${ }^{++}$two missing observations

${ }^{+++}$three missing observations 


\section{Supplementary table B:}

\section{Logistic regression}

\begin{tabular}{lcc}
\hline Variable & OR $^{\#}$ & $\mathbf{9 5 \%}$ CI \\
28 Tender joint & $1.13^{\#}$ & 1.02 to 1.25 \\
SF36-MCS & $0.93^{\#}$ & 0.88 to 0.99 \\
HAQ-DI & 2.01 & 0.80 to 5.06 \\
CRP $^{\S} \mathrm{mg} / \mathrm{mL}$ & $0.41^{*}$ & 0.16 to 1.04 \\
Female sex $^{\alpha}$ & 1.92 & 0.42 to 8.74 \\
Age $^{\alpha}$ & 1.00 & 0.97 to 1.03 \\
Disease duration $^{\not}$ & 1.00 & 0.99 to 1.01 \\
Initiated bDMARD $^{\alpha}$ & 2.47 & 0.68 to 8.97 \\
\hline
\end{tabular}

All variables were included as continuous scores, except 'female sex' and 'initiated bDMARD'.

${ }^{\alpha}$ Pre-specified confounder to be included in the adjusted model.

${ }^{\S} \mathrm{CRP}$ was logtransformed in the analyses.

$* \mathrm{p}<0.1$

${ }^{\#} \mathrm{p}<0.05$ 